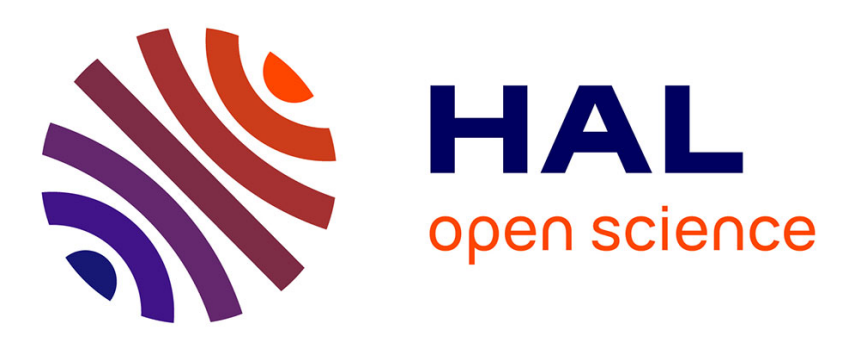

\title{
From exact observability to identification of singular sources
}

\author{
Marius Tucsnak, George Weiss
}

\section{To cite this version:}

Marius Tucsnak, George Weiss. From exact observability to identification of singular sources. Mathematics of Control, Signals, and Systems, 2015, 27 (1), pp.1-21. 10.1007/s00498-014-0132-z . hal01278500

\section{HAL Id: hal-01278500 \\ https://hal.science/hal-01278500}

Submitted on 24 Feb 2016

HAL is a multi-disciplinary open access archive for the deposit and dissemination of scientific research documents, whether they are published or not. The documents may come from teaching and research institutions in France or abroad, or from public or private research centers.
L'archive ouverte pluridisciplinaire HAL, est destinée au dépôt et à la diffusion de documents scientifiques de niveau recherche, publiés ou non, émanant des établissements d'enseignement et de recherche français ou étrangers, des laboratoires publics ou privés. 


\title{
From exact observability to identification of singular sources
}

\author{
Marius Tucsnak \\ Department of Mathematics \\ University of Nancy \\ Vandoeuvre les Nancy 54506, France \\ Marius.Tucsnak@iecn.u-nancy.fr
}

\author{
George Weiss \\ Department of EE-Systems \\ Tel Aviv University \\ Ramat Aviv 69978, Israel \\ gweiss@eng.tau.ac.il
}

\begin{abstract}
We give general results about the identifiability of source terms for infinite dimensional linear systems that are exactly observable. We allow the source term to be unbounded, i.e., not contained in the state space, but in one of a sequence of extended spaces. We show that the operator from the source term to the output function is bounded from below, in suitable norms. We apply the main result to a system described by the wave equation in a bounded $n$-dimensional domain. We derive results of independent interest concerning the range of the input map of an exactly controllable system, when restricted to various spaces of smooth input functions.
\end{abstract}

\section{Introduction}

In this work we give some general results about the identifiability of source terms for infinite dimensional linear systems that are exactly observable. We allow the source term to be unbounded, i.e., not contained in the state space. The abstract main result (presented in Section 4) is a generalization of the main result of Alves et al [1], which in turn is inspired by Puel and Yamamoto [17]. The class of source terms that we consider is larger than in [1], which makes it possible to tackle a large range of applications to systems governed by partial differential equations. In the applications, the general aim is to show that the location of point sources can be determined from the output function in a continuous manner (in other words, we have a stability estimate for the considered inverse problem). For the physical background of such problems see our references.

Our motivating example is a wave equation with a point source and Neumann boundary observation on a part of the boundary:

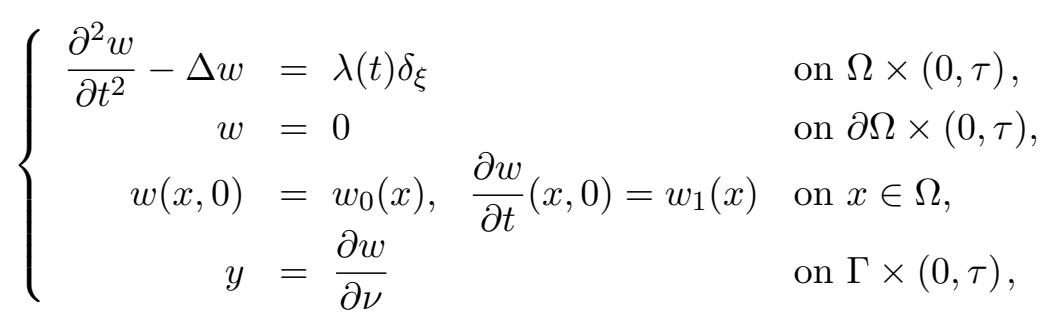

where $\Omega \subset \mathbb{R}^{n}$ is a bounded domain with $C^{2}$ boundary, $\tau>0, \lambda$ is a given absolutely continuous function with $\lambda^{\prime} \in L^{1}[0, \tau]$ and $\lambda(0) \neq 0$. The distribution $\delta_{\xi}$ is the Dirac 
mass located at $\xi \in \Omega$ and $\Gamma$ is a nonempty open subset of $\partial \Omega$. The initial data satisfy $w_{0} \in \mathcal{H}_{0}^{1}(\Omega)$ and $w_{1} \in L^{2}(\Omega)$ and they are known. The well-posedness of the system (1.1) has been investigated for $n \in\{1,2,3\}$ in Triggiani [18, Theorem 2.1] (see also Meyer [16] for the case $n=3$ and without the boundary observation).

The inverse problem is to determine $\xi$ from $y$ in a continuous way. In fact, we may replace in this problem the distribution $\delta_{\xi}$ with a finite sum of the type $\sum_{k=1}^{N} \delta_{\xi_{k}}$, with $\xi_{k} \in \Omega$, without essentially changing the difficulty of the problem, and then the problem is to determine all the points $\xi_{k}$. To keep the exposition simple, in this Introduction we stick to a single Dirac mass at $\xi$. This problem (with a finite sum of Dirac masses) has been considered by Komornik and Yamamoto [12, Section 4] under the assumptions that $\Omega$ is a ball in $\mathbb{R}^{n}$ and $\Gamma$ is its whole boundary. There is a large engineering literature on related localization problems with point sources and point measurements, see for instance A.J. Weiss [21] and the references therein.

We say that the wave equation on $\Omega$ with the Neumann trace operator on $\Gamma$ is exactly observable in time $\tau_{0}>0$ if the following estimate holds for smooth solutions of (1.1) with $\lambda=0$ :

$$
\|y\|_{L^{2}\left(\left[0, \tau_{0}\right] ; L^{2}(\Gamma)\right)} \geqslant k\left(\left\|\nabla w_{0}\right\|_{L^{2}(\Omega)}^{2}+\left\|w_{1}\right\|_{L^{2}(\Omega)}^{2}\right)^{\frac{1}{2}},
$$

where $k>0$. We refer to [20, Chapter 7] for more details on this concept. If $\partial \Omega$ is sufficiently smooth, then the above observability property is equivalent to the geometric optics condition due to Bardos, Lebeau and Rauch [2] (see also Burq and Gérard [3]).

For any Hilbert space $U$, any $m \in \mathbb{N}$ and any $\tau>0$ we set

$$
\begin{aligned}
& \mathcal{H}_{L}^{m}(0, \tau ; U)=\left\{u \in \mathcal{H}^{m}(0, \tau ; U) \mid u(0)=\cdots=u^{m-1}(0)=0\right\}, \\
& \mathcal{H}_{R}^{m}(0, \tau ; U)=\left\{u \in \mathcal{H}^{m}(0, \tau ; U) \mid u(\tau)=\cdots=u^{m-1}(\tau)=0\right\} .
\end{aligned}
$$

For $m=0$ the above spaces are defined to be $L^{2}([0, \tau] ; U)$. We denote, as is the standard practice, $\mathcal{H}_{0}^{m}(0, \tau ; U)=\mathcal{H}_{L}^{m}(0, \tau ; U) \cap \mathcal{H}_{R}^{m}(0, \tau ; U)$. We denote by $W^{1,1}(0, \tau)$ the space of absolutely continuous functions on the interval $(0, \tau)$ whose derivative is in $L^{1}[0, \tau]$.

Our main result, Theorem 4.4 requires some preliminaries, so we state here only its consequences when applied to the above wave equation with a point source term.

Theorem 1.1. Assume that the wave equation on $\Omega$ with the Neumann trace operator on $\Gamma$ is exactly observable in time $\tau_{0}>0$. Let $\varepsilon>0$ be such that the open set

$$
\Omega_{\varepsilon}=\{x \in \Omega \mid \operatorname{dist}(x, \partial \Omega)>\varepsilon\}
$$

is not empty. Let $m \in \mathbb{N}$ be such that $m>n / 2$ and let $\tau>\tau_{0}$. Let $\lambda \in W^{1,1}(0, \tau)$.

Then there exists a constant $K_{\varepsilon, m, \tau}>0$ (which depends also on $\lambda$ ) with the following property: if $y^{(1)}$ and $y^{(2)}$ are the outputs of the system (1.1) corresponding to $\xi=\xi^{(1)} \in \Omega_{\varepsilon}$ and $\xi=\xi^{(2)} \in \Omega_{\varepsilon}$, respectively, with the same initial data, then

$$
\left|\xi^{(1)}-\xi^{(2)}\right| \leqslant K_{\varepsilon, m, \tau}\left\|y^{(1)}-y^{(2)}\right\|_{\left[\mathcal{H}_{R}^{m-1}\left(0, \tau ; L^{2}(\Gamma)\right)\right]^{\prime}} .
$$

Here $\left[\mathcal{H}_{R}^{m-1}\left(0, \tau ; L^{2}(\Gamma)\right)\right]^{\prime}$ is the dual of $\mathcal{H}_{R}^{m-1}\left(0, \tau ; L^{2}(\Gamma)\right)$ with respect to the pivot space $L^{2}\left([0, \tau] ; L^{2}(\Gamma)\right)$, and $|x|$ denotes the Euclidean norm of $x \in \mathbb{R}^{n}$. For the proof of this theorem we refer to Section 5 . 
The theorem tell us that we can recover $\xi$ from $y$ in a continuous way, with respect to the specified norm on the output functions. Of course, the $\left[\mathcal{H}_{R}^{m}\right]^{\prime}$ norm decreases as $m$ increases, so that formally we have stronger statements for larger $m$, but in applications it is probably enough to take one integer $m$ that satisfies $m>n / 2$. For $m \leqslant n / 2$ the right-hand side of (1.2) may become meaningless, because $y^{(1)}, y^{(2)}$ may not be in the required space (whose norm we are using).

This theorem is a generalization of the result in [12, Section 4], in the sense that in [12] $\Omega$ is restricted to be a ball. On the other hand, for a ball, the result in [12, Section 4] is more general than ours in the sense that they consider related estimates for all $m \geqslant \frac{n+1}{2}$ (not necessarily integer). Moreover, in [12], the left side of (1.2) appears at a positive power, so that the estimates are not equivalent. While the analysis in [12] is based on series expansions into spherical functions, we use an abstract result given in Section 4 . This abstract result can be applied also to other examples, for example, systems described by the heat, plate or Schrödinger equation.

An interesting related problem is to determine both the localization $\xi$ and the intensity $\lambda$ from boundary measurements. This is, in the general case, an open problem. Some results in this direction can be found in El Badia and Ha-Duong [6] and [1, Section 6].

To prove our abstract result from Section 4, we need some new results about exactly controllable systems: the range of the input map when restricted to inputs in certain smooth Sobolev spaces. These new results are derived in Section 2 (and the dual results in Section 3). We believe that these sections are of independent interest.

\section{Controllability with smooth inputs for smooth final states}

For linear infinite-dimensional systems, exact controllability in some time $\tau$ means that, starting from the initial state zero, with a suitably chosen input function $u$ of class $L^{2}$, we can reach any final state at time $\tau$. This property and its dual, exact observability, have been discussed in a very large number of papers and books, many inspired by the book Lions [15]. Relatively few authors have considered the following question: if the desired final state is in some smoother subspace, for example, the domain of the generator to some power, can the corresponding input function also be chosen in a smoother subspace, for example, a Sobolev space with positive index. A systematic study of this question has been undertaken in a recent paper by Ervedoza and Zuazua [7]. For the specific case of the wave equation with bounded (distributed) control, it was considered in Dehman and Lebeau [5]. There are several related results in Section 11.3 of [20]. Here we derive more results on this topic, that generalize certain results from [7] and [20].

Throughout this section, $X$ and $U$ are Hilbert spaces.

Our first standing assumption for this section is that $\mathbb{T}$ is a strongly continuous semigroup of operators on $X$, with generator $A: \mathcal{D}(A) \rightarrow X$. For $m \in \mathbb{N}$, we denote $X_{m}=\mathcal{D}\left(A^{m}\right)$ and $X_{m}^{d}=\mathcal{D}\left(A^{* m}\right)$, each with the graph norm, so that they are Hilbert spaces too. We set $X_{0}=X_{0}^{d}=X$. We denote

$$
X_{-m}=\left(X_{m}^{d}\right)^{\prime}, \quad X_{-m}^{d}=\left(X_{m}\right)^{\prime},
$$

all these dual spaces being with respect to the pivot space $X$. Note that, for each $k \in \mathbb{Z}$, the original semigroup $\mathbb{T}$ has a restriction (or an extension) to $X_{k}$ that is the image of $\mathbb{T}$ 
through the unitary operator $(\beta I-A)^{-k} \in \mathcal{L}\left(X, X_{k}\right)$, where $\beta \in \rho(A)$ (the resolvent set of $A)$. We refer to [20, Remark 2.10.5] for a proof of the last statement.

The second standing assumption for this section is that $B \in \mathcal{L}\left(U, X_{-1}\right)$ is an admissible control operator for $\mathbb{T}$. The admissibility assumption means that for some $\tau>0$, the operator $\Phi_{\tau}$ defined by

$$
\Phi_{\tau} u=\int_{0}^{\tau} \mathbb{T}_{\tau-\sigma} B u(\sigma) \mathrm{d} \sigma \quad \forall u \in L^{2}([0, \tau] ; U),
$$

a priori with range Ran $\Phi_{\tau}$ contained in $X_{-1}$, has its range contained in $X$. We refer again to [20] for more material on this concept. Here we only mention that it follows from the admissibility assumption that $\Phi_{\tau} \in \mathcal{L}\left(L^{2}([0, \tau] ; U), X\right)$ holds for all $\tau \geqslant 0$, and (with $u$ as above) $\Phi_{t} u$ depends continuously on $t \in[0, \tau]$. The operators $\Phi_{\tau}$ are called input maps corresponding to the pair $(A, B)$.

Lemma 2.1. If $u \in \mathcal{H}_{L}^{m}(0, \tau ; U)$ (with $\left.m \in \mathbb{N}\right)$ and $z(t)=\Phi_{t} u$ for every $t \in[0, \tau]$, then

$$
z \in C^{m}([0, \tau] ; X), \quad z^{(j)}(0)=0 \quad \forall j \in\{0,1, \ldots m\}
$$

and, for all $t \in[0, \tau]$,

$$
z^{(m)}(t)=A z^{(m-1)}(t)+B u^{(m-1)}(t)
$$

Proof. The case $m=1$ is contained in Proposition 4.2.5 and Lemma 4.2.8 of [20]. Now suppose that the statement is true for $m-1$, i.e., for $u \in \mathcal{H}_{L}^{m-1}(0, \tau ; U)$ we have

$$
z \in C^{m-1}([0, \tau] ; X), \quad z^{(j)}(0)=0 \quad \forall j \in\{0,1, \ldots m-1\}
$$

and, for all $t \in[0, \tau]$,

$$
z^{(m-1)}(t)=A z^{(m-2)}(t)+B u^{(m-2)}(t) .
$$

Suppose that $u \in \mathcal{H}_{L}^{m}(0, \tau ; U)$, so that $B u^{(m-2)} \in \mathcal{H}_{L}^{2}\left(0, \tau ; X_{-1}\right) \subset C^{1}\left([0, \tau] ; X_{-1}\right)$. It is clear from (2.3) that also $A z^{(m-2)} \in C^{1}\left([0, \tau] ; X_{-1}\right)$. Thus, (2.4) implies that $z^{(m-1)} \in$ $C^{1}\left([0, \tau] ; X_{-1}\right)$. Differentiating both sides of $(2.4)$ we obtain that $(2.2)$ holds as an equality in $X_{-1}$, for all $t \in[0, \tau]$. In particular, for $t=0$ we obtain that $z^{(m)}(0)=0$.

Denote $x=z^{(m-1)}$ and $v=u^{(m-1)}$, so that $x \in C^{1}\left([0, \tau] ; X_{-1}\right), v \in \mathcal{H}_{L}^{1}(0, \tau ; U)$ and (according to (2.2)) $\dot{x}=A x+B v$ holds on $[0, \tau]$ and $x(0)=0$. According to Proposition 4.2.5 in [20] we have $x(t)=\Phi_{t} v$ for every $t \in[0, \tau]$ and according to Lemma 4.2.8 in [20] we have $x \in C^{1}([0, \tau] ; X)$. This implies that $z \in C^{m}([0, \tau] ; X)$ (so that and (2.2) holds as an equality in $X)$. Thus, by induction, we have proved the lemma.

Lemma 2.2. For every $m \in \mathbb{N}$ define the space $Z_{m} \subset X$ by

$$
Z_{m}=X_{m}+(\beta I-A)^{-1} B U+(\beta I-A)^{-2} B U \ldots+(\beta I-A)^{-m} B U,
$$

where $\beta \in \rho(A)$. We set $Z_{0}=X$. Then $Z_{m}$ is independent of the choice of $\beta \in \rho(A)$.

Proof. For brevity, in this proof we denote $R_{\mu}=(\mu I-A)^{-1}$. Let $\beta$ be the number used in (2.5). We break the proof into a sequence of claims.

Claim 0. We have $Z_{m} \subset Z_{m-1}$, for all $m \in \mathbb{N}$. This is easy to see.

Claim 1. We have $R_{\beta} Z_{m-1} \subset Z_{m}$, for all $m \in \mathbb{N}$. This is obvious. 
Claim 2. For any $\mu \in \rho(A)$ we have $R_{\mu} B U \subset Z_{m}$, for all $m \in \mathbb{N}$. This is shown by induction. It is obviously true for $m=0$. Suppose it is true for $m-1$, for some $m \in \mathbb{N}$. From the resolvent identity we have

$$
R_{\mu} B U \subset R_{\beta} B U+R_{\beta} R_{\mu} B U \subset Z_{m}+R_{\beta} Z_{m-1} .
$$

Using Claim 1, we obtain that $R_{\mu} B U \subset Z_{m}$.

Claim 3. We have the recurrence relation

$$
Z_{m}=R_{\beta}\left[Z_{m-1}+B U\right]
$$

for all $m \in \mathbb{N}$. This is easy to see.

Claim 4. We have $R_{\mu} Z_{m-1} \subset Z_{m}$, for all $m \in \mathbb{N}$ and for all $\mu \in \rho(A)$. This is proved by induction. The case $m=1$ is obvious. Suppose that the claim holds for some $m \in \mathbb{N}$. Applying $R_{\mu}$ to the recurrence relation in Claim 3 and then using Claim 2, we get

$$
R_{\mu} Z_{m}=R_{\beta} R_{\mu} Z_{m-1}+R_{\beta} R_{\mu} B U \subset R_{\beta} Z_{m}+R_{\beta} Z_{m} .
$$

Finally, using Claim 1 we get $R_{\mu} Z_{m} \subset Z_{m+1}$, i.e., the claim holds for $m+1$.

Claim 5. For some $\mu \in \rho(A)$ and all $m \in \mathbb{N}$ define

$$
\tilde{Z}_{m}=X_{m}+R_{\mu} B U+R_{\mu}^{2} B U \ldots+R_{\mu}^{m} B U .
$$

Then $\tilde{Z}_{m} \subset Z_{m}$. Indeed, the first term $X_{m}$ in the above sum is obviously a subset of $Z_{m}$. For the other terms we have, using Claim 2 and then repeatedly Claims 0 and 4, that for every $k \in \mathbb{N}$,

$$
R_{\mu}^{k} B U \subset R_{\mu}^{k-1} R_{\mu} B U \subset R_{\mu}^{k-1} Z_{m} \subset Z_{m} .
$$

Claim 6. We have $\tilde{Z}_{m}=Z_{m}$, for all $m \in \mathbb{N}$. Indeed, by redoing the above steps with the roles of $\beta$ and $\mu$ reversed, we obtain that $Z_{m} \subset \tilde{Z}_{m}$.

Proposition 2.3. For every $m \in\{0,1,2, \ldots\}$ and for all $\tau>0$,

$$
\Phi_{\tau} \mathcal{H}_{L}^{m}(0, \tau ; U) \subset Z_{m}, \quad \Phi_{\tau} \mathcal{H}_{0}^{m}(0, \tau ; U) \subset X_{m},
$$

where $Z_{m}$ is the space introduced in Lemma 2.2 .

Proof. It is well known that if $\beta \in \mathbb{C}$ then $B$ is admissible also for the semigroup generated by $A-\beta I$. It is easy to see that the range of the operator $\Phi_{\tau}$, when applied to one of the spaces $\mathcal{H}_{L}^{m}(0, \tau ; U)$ or $\mathcal{H}_{0}^{m}(0, \tau ; U)$, does not change if we replace $A$ with $A-\beta I$. For these reasons, without loss of generality we may assume in this proof that $\mathbb{T}$ is exponentially stable. Therefore we may take $\beta=0$ in (2.5).

Let $u \in \mathcal{H}_{L}^{m}(0, \tau ; U)$ and let $z:[0, \tau] \rightarrow X$ be defined by $z(t)=\Phi_{t} u$. We know from Lemma 2.1 that (2.1) and (2.2) hold, for any $k \in \mathbb{N}$ with $k \leqslant m$ in place of $m$. Applying $A^{-1}$ to both sides of $(2.2)$ we obtain that (for $1 \leqslant k \leqslant m$ )

$$
z^{(k-1)}(\tau)=A^{-1} z^{(k)}(\tau)-A^{-1} B u^{(k-1)}(\tau) .
$$

Substituting the case $k=2$ into the case $k=1$, then substituting the case $k=3$ into the resulting formula and so on, we obtain that

$$
z(\tau)=A^{-m} z^{(m)}(\tau)-A^{-1} B u(\tau)-A^{-2} B u^{(1)}(\tau) \ldots-A^{-m} B u^{(m-1)}(\tau) .
$$


According to (2.5) we get $z(\tau) \in Z_{m}$. If we assume that $u \in \mathcal{H}_{0}^{m}(0, \tau ; U)$ then all the terms containing $u$ on the right-hand side of (2.6) vanish, so that we get $z(\tau) \in X_{m}$.

The third standing assuption that will be used in the remaining part of this section is that the pair $(A, B)$ is exactly controllable in some time $\tau_{0}>0$. This ssumption means that $\operatorname{Ran} \Phi_{\tau_{0}}=X$ (when using inputs of class $L^{2}$ ). In this case, Ran $\Phi_{\tau}=X$ holds for all $\tau>\tau_{0}$, as it is easy to see. The reader may look up, for instance, [20, Chapter 11] for some consequences of this concept, equivalent conditions and examples.

Lemma 2.4. Assume that $\mathbb{T}$ is exponentially stable. Then there exists a constant $c>0$ with the following property: For any $\lambda>0$ consider the two systems with states $w(t) \in X$ and $u(t) \in U$ and the common input function $v$, described by

$$
\dot{w}=A w+B v, \quad \dot{u}=\lambda u+v .
$$

These systems are simultaneously exactly controllable in any time

$$
\tau>\tau_{0}+\frac{c}{\lambda}
$$

This means that for any $w_{0} \in X$ and any $u_{0} \in U$, there exists $v \in L^{2}([0, \tau] ; U)$ such that the solutions $w, u$ of $(2.7)$ corresponding to $w(0)=0$ and $u(0)=0$ satisfy

$$
w(\tau)=w_{0}, \quad u(\tau)=u_{0} .
$$

The dual (and hence equivalent) version of this lemma (with some additional information on the constant $c$ ) has appeared as Lemma 11.3.5 in [20], so that we omit the proof. If $U$ is finite-dimensional, then a slightly stronger conclusion (corresponding to $c=0$ in (2.8)) can be obtained from [19, Theorem 3.3] (see also [20, Corollary 11.3.3]).

Theorem 2.5. For every $m \in\{0,1,2, \ldots\}$ and for all $\tau>\tau_{0}$,

$$
\Phi_{\tau} \mathcal{H}_{L}^{m}(0, \tau ; U)=Z_{m}, \quad \Phi_{\tau} \mathcal{H}_{0}^{m}(0, \tau ; U)=X_{m} .
$$

Proof. We prove the theorem using induction. The case $m=0$ is true by the definition of exact controllability ( since $Z_{0}=X_{0}=X$ ). Now suppose that the theorem is true for $m-1$, where $m \in \mathbb{N}$. We have to prove that the theorem is true for $m$. While proving this, we may assume, without loss of generality, that $\mathbb{T}$ is exponentially stable (this was explained at the beginning of the proof of Proposition 2.3). In view of Proposition 2.3, we only have to prove the converse inclusions.

The proof of $\Phi_{\tau} \mathcal{H}_{L}^{m}(0, \tau ; U) \supset Z_{m}$ :

Choose $\tau>\tau_{0}$ and then choose $\lambda>0$ such that (2.8) holds. Consider the two systems with states $w(t) \in X$ and $u(t) \in U$ and the common input function $v$, described by (2.7). According to Lemma 2.4, these systems are simultaneously exactly controllable in time $\tau$. This means that the combined system described by $\dot{q}=\mathcal{A} q+\mathcal{B} v$, with

$$
\mathcal{A}=\left[\begin{array}{cc}
A & 0 \\
0 & \lambda I
\end{array}\right], \quad \mathcal{B}=\left[\begin{array}{c}
B \\
I
\end{array}\right]
$$

with state space $\mathcal{X}=X \times U$, is exactly controllable in time $\tau$. Let us denote by $\tilde{\Phi}_{\tau}$ the input to state operator for the combined system, given by

$$
\tilde{\Phi}_{\tau}=\left[\begin{array}{c}
\Phi_{\tau} \\
\varphi_{\tau}
\end{array}\right], \quad \text { where } \quad \varphi_{\tau} v=\int_{0}^{\tau} e^{\lambda(\tau-\sigma)} v(\sigma) \mathrm{d} \sigma .
$$


According to the theorem applied for $m-1$, we have

$$
\tilde{\Phi}_{\tau} \mathcal{H}_{L}^{m-1}(0, \tau ; U)=\mathcal{Z}_{m-1},
$$

where $\mathcal{Z}_{m-1}$ is defined similarly as in $(2.5)$, so that

$$
\mathcal{Z}_{m-1}=Z_{m-1} \times U \text {. }
$$

For an arbitrary $z^{0} \in Z_{m}$ choose $w^{0} \in Z_{m-1}, u^{0} \in U$ such that

$$
z^{0}=A^{-1}\left[w^{0}-B u^{0}\right] .
$$

Indeed, this is possible according to the recurrence relation in Claim 3 of the proof of Lemma 2.2. From (2.10) there exists an input signal $v \in \mathcal{H}_{L}^{m-1}(0, \tau ; U)$ such that the solutions $w, u$ of (2.7) satisfy

$$
w(0)=0, \quad w(\tau)=w^{0}-\lambda z^{0}, \quad u(0)=0, u(\tau)=u^{0} .
$$

Moreover, it is easy to see that $u \in \mathcal{H}_{L}^{m}(0, \tau ; U)$. We define $z \in C([0, \tau] ; X)$ by

$$
z(t)=(A-\lambda I)^{-1}[w(t)-B u(t)] .
$$

It is clear that $z(0)=0$. It is easy to see that

$$
z(\tau)=(A-\lambda I)^{-1}\left[w^{0}-B u^{0}-\lambda z^{0}\right]=(A-\lambda I)^{-1}\left[A z^{0}-\lambda z^{0}\right]=z^{0} .
$$

This part of the proof will be complete if we show that $z$ is a solution of

$$
\dot{z}(t)=A z(t)+B u(t),
$$

so that $z(\tau)=\Phi_{\tau} u$. First we verify that $z$ satisfies the differential equation

$$
\dot{z}(t)=\lambda z(t)+w(t) \quad \forall t \in[0, \tau] .
$$

Indeed, we have (using the definition of $z$ )

$$
\begin{aligned}
\dot{z}(t) & =(A-\lambda I)^{-1}[\dot{w}(t)-B \dot{u}(t)]=(A-\lambda I)^{-1}[A w(t)-\lambda B u(t)] \\
& =(A-\lambda I)^{-1}[(A-\lambda I) w(t)+\lambda(w(t)-B u(t))]=w(t)+\lambda z(t) .
\end{aligned}
$$

Note that $(2.13)$ implies that $z \in C^{1}([0, \tau] ; X)$. Now from (2.13) we get, using again the definition of $z$,

$$
\begin{aligned}
\dot{z}(t) & =(\lambda I-A+A)(A-\lambda I)^{-1}[w(t)-B u(t)]+w(t) \\
& =-[w(t)-B u(t)]+A(A-\lambda I)^{-1}[w(t)-B u(t)]+w(t) \\
& =A z(t)+B u(t) .
\end{aligned}
$$

The proof of $\Phi_{\tau} \mathcal{H}_{0}^{m}(0, \tau ; U) \supset X_{m}$ :

This part of the proof is very similar to the previous part. The difference is that now instead of (2.10) we use

$$
\tilde{\Phi}_{\tau} \mathcal{H}_{0}^{m-1}(0, \tau ; U)=\mathcal{X}_{m-1},
$$


where $\mathcal{X}_{m-1}=\mathcal{D}\left(\mathcal{A}^{m-1}\right)=\mathcal{D}\left(A^{m-1}\right) \times U$. This time we choose $u^{0}=0, v \in \mathcal{H}_{0}^{m-1}(0, \tau ; U)$ and we obtain that $u \in \mathcal{H}_{0}^{m}(0, \tau ; U)$.

We remark that the equality $\Phi_{\tau} \mathcal{H}_{L}^{1}(0, \tau ; U)=Z_{1}$ (which follows from the above theorem) constitutes Theorem 11.3.6 in [20]. The equality $\Phi_{\tau} \mathcal{H}_{L}^{2}(0, \tau ; U)=Z_{2}$, for finitedimensional $U$, constitutes Proposition 11.3.8 in [20]. Our proof above uses ideas from the proofs of the results cited. The inclusion $\Phi_{\tau} \mathcal{H}_{0}^{m}(0, \tau ; U) \supset X_{m}$ is contained in Theorem 1.4 of [7], but under the additional assumption that the semigroup $\mathbb{T}$ is invertible.

We define a norm on the space $Z_{m}$ from (2.5) as follows:

$$
\|z\|_{Z_{m}}^{2}=\inf \left\{\|x\|_{m}^{2}+\left\|u_{1}\right\|^{2}+\left\|u_{2}\right\|^{2} \ldots+\left\|u_{m}\right\|^{2}\right\},
$$

the infimum being computed over all the collections $\left(x, u_{1}, u_{2}, \ldots u_{m}\right) \in X_{m} \times U^{m}$ such that $z=x+(\beta I-A)^{-1} B u_{1}+(\beta I-A)^{-2} B u_{2} \ldots+(\beta I-A)^{-m} B u_{m}$. Then $Z_{m}$ can be regarded as a subspace of $X_{m} \times U^{m}$, namely, the orthogonal complement of the space

$$
\mathcal{N}=\left\{\left(\left(x, u_{1}, u_{2}, \ldots u_{m}\right) \in X_{m} \times U^{m} \mid x+\sum_{k=1}^{m}(\beta I-A)^{-k} B u_{k}=0\right\} .\right.
$$

This shows that $Z_{m}$ is complete (hence, it is a Hilbert space). It is easy to see that for all $m \in \mathbb{N}$ the embeddings

$$
X_{m} \subset Z_{m} \subset Z_{m-1}
$$

are continuous. (For $m=1$ see [20, p. 119].) Usually, $X_{m}$ is not dense in $Z_{m}$.

\section{Extensions of output maps}

In this section we formulate and prove the dual versions of the results from Section 2 and we give a very simple example. Throughout this section, $X$ and $Y$ are Hilbert spaces.

Our first standing assumption for this section is that $\mathbb{T}$ is a strongly continuous semigroup of operators on $X$, with generator $A: \mathcal{D}(A) \rightarrow X$. For $m \in \mathbb{Z}$, the spaces $X_{m}$, $X_{m}^{d}, X_{-m}$ and $X_{-m}^{d}$ are defined as in Section 2.

The second standing assumption for this section is that $C \in \mathcal{L}\left(X_{1}, Y\right)$ is an admissible observation operator for $\mathbb{T}$. The admissibility assumption means that for some $\tau>0$, the operator $\Psi_{\tau}$ defined by

$$
\left(\Psi_{\tau} z_{0}\right)(t)=C \mathbb{T}_{t} z_{0} \quad \forall z_{0} \in X_{1},
$$

has an extension to an operator $\Psi_{\tau} \in \mathcal{L}\left(X, L^{2}([0, \tau], Y)\right.$. Equivalently, there is a positive number $k$ such that $\int_{0}^{\tau}\left\|C \mathbb{T}_{t} z_{0}\right\|^{2} \mathrm{~d} t \leqslant k^{2}\left\|z_{0}\right\|^{2}$ for all $z_{0} \in \mathcal{D}(A)$. We refer to $[20,23]$ for more material on this concept. Here we only mention that it follows from the admissibility assumption that $\Psi_{\tau} \in \mathcal{L}\left(X, L^{2}([0, \tau] ; Y)\right.$ holds for all $\tau \geqslant 0$. The operators $\Psi_{\tau}$ are called output maps corresponding to the pair $(A, C)$.

The dual result of Proposition 2.3 is the following:

Proposition 3.1. For every $m \in\{0,1,2, \ldots\}$ and for all $\tau>0, \Psi_{\tau}$ has unique extensions

$$
\Psi_{\tau} \in \mathcal{L}\left(\left(Z_{m}^{d}\right)^{\prime},\left[\mathcal{H}_{R}^{m}(0, \tau ; Y)\right]^{\prime}\right), \quad \Psi_{\tau} \in \mathcal{L}\left(\left(X_{-m}, \mathcal{H}^{-m}(0, \tau ; Y)\right),\right.
$$


where

$$
Z_{m}^{d}=X_{m}^{d}+\left(\beta I-A^{*}\right)^{-1} C^{*} U+\left(\beta I-A^{*}\right)^{-2} C^{*} U \ldots+\left(\beta I-A^{*}\right)^{-m} C^{*} U,
$$

for some $\beta \in \rho\left(A^{*}\right)$. The norm on $Z_{m}^{d}$ is defined similarly as in $(2.14)$, with $A^{*}$ and $C^{*}$ in place of $A$ and $B$. Here and below, dualities are computed with respect to the pivot spaces $X$ and $L^{2}([0, \tau] ; Y)$, respectively.

Proof. Denote by $\Phi_{\tau}^{d}$ the input maps corresponding to the pair $\left(A^{*}, C^{*}\right)$. We know from $[20$, Section 4.4] that

$$
\Phi_{\tau}^{d}=\Psi_{\tau}^{*} \boldsymbol{Я}_{\tau},
$$

where $\boldsymbol{Я}_{\tau}$ is the reflection operator on $L^{2}([0, \tau] ; Y)$ defined by $\boldsymbol{Я}_{\tau} u(t)=u(\tau-t)$. Notice that $\boldsymbol{\Omega}_{\tau}$ is self-adjoint and also unitary. Since $\boldsymbol{\Omega}_{\tau}$ is an isomorphism from $\mathcal{H}_{L}^{m}(0, \tau ; Y)$ to $\mathcal{H}_{R}^{m}\left(0, \tau ; Y\right.$ ), it follows from Proposition 2.3 (with $A^{*}$ and $C^{*}$ in place of $A$ and $B$ ) that

$$
\Psi_{\tau}^{*} \mathcal{H}_{R}^{m}(0, \tau ; Y) \subset Z_{m}^{d}, \quad \Psi_{\tau}^{*} \mathcal{H}_{0}^{m}(0, \tau ; Y) \subset X_{m}^{d} .
$$

where $Z_{m}^{d}$ is the counterpart of $Z_{m}$ for the pair $\left(A^{*}, C^{*}\right)$, so that it is given by (3.2). By the closed graph theorem we have that $\Psi_{\tau}^{*}$ is bounded between the spaces indicated above. Now taking adjoints we obtain (3.1).

The third standing assuption for the remaining part of this section is that the pair $(A, C)$ is exactly observable in some time $\tau_{0}>0$. This assumption means that $\Psi_{\tau_{0}}$ is bounded from below. In this case, $\Psi_{\tau}$ is bounded from below for all $\tau>\tau_{0}$, as it is easy to see. We refer to [20, Chapter 6] for more material on this concept.

The dual result of Theorem 2.5 is the following:

Theorem 3.2. With the notation of Proposition 3.1, for every $m \in\{0,1,2, \ldots\}$ and each $\tau>\tau_{0}$, there exists a constant $c_{m, \tau}>0$ such that, for every $f \in\left(Z_{m}^{d}\right)^{\prime}$, we have

$$
\left\|\Psi_{\tau} f\right\|_{\left[\mathcal{H}_{R}^{m}(0, \tau ; Y)\right]^{\prime}} \geqslant c_{m, \tau}\|f\|_{\left(Z_{m}^{d}\right)^{\prime}}
$$

Similarly, for every $m \in\{0,1,2, \ldots\}$ and each $\tau>\tau_{0}$, there exists a constant $k_{m, \tau}>0$ such that, for every $f \in X_{-m}$, we have

$$
\left\|\Psi_{\tau} f\right\|_{\mathcal{H}^{-m}(0, \tau ; Y)} \geqslant k_{m, \tau}\|f\|_{X_{-m}} .
$$

Proof. We denote again by $\Phi_{\tau}^{d}$ the input maps corresponding to the pair $\left(A^{*}, C^{*}\right)$. We know from [20, Theorem 11.2.1] that $\left(A^{*}, C^{*}\right)$ is exactly controllable in time $\tau_{0}$. According to Theorem 2.5 (applied to the pair $\left(A^{*}, C^{*}\right)$ ), for every $m \in\{0,1,2, \ldots\}$ and for all $\tau>\tau_{0}$, $\Phi_{\tau}^{d}$ maps $\mathcal{H}_{L}^{m}(0, \tau ; Y)$ onto $Z_{m}^{d}$, and it also maps $\mathcal{H}_{0}^{m}(0, \tau ; Y)$ onto $X_{m}^{d}$. Since $\Psi_{\tau}^{*}=\Phi_{\tau}^{d} \boldsymbol{g}_{\tau}$ (see (3.3)), it follows that

$$
\Psi_{\tau}^{*} \mathcal{H}_{R}^{m}(0, \tau ; Y)=Z_{m}^{d}, \quad \Psi_{\tau}^{*} \mathcal{H}_{0}^{m}(0, \tau ; Y)=X_{m}^{d} .
$$

By the closed graph theorem, $\Psi_{\tau}^{*}$ is bounded between these spaces. By a well known result about surjective operators (see, for instance, [20, Proposition 12.1.3]), $\Psi_{\tau}$ is bounded from below between the corresponding dual spaces. This fact is expressed in (3.4) and (3.5).

Example 3.3. Consider the boundary observed wave equation on the interval $(0, \pi)$ :

$$
w_{t t}=w_{x x}, \quad w(0, t)=w(\pi, t)=0,
$$


with the initial conditions

$$
w(x, 0)=w_{0}(x), \quad w_{t}(x, 0)=w_{1}(x),
$$

and with the measurements

$$
y_{1}(t)=-\frac{\partial w}{\partial x}(0, t), \quad y_{2}(t)=\frac{\partial w}{\partial x}(\pi, t) .
$$

We refer to Section 5 for an $n$-dimensional version of this system.

To associate to these equations a system in the sense considered earlier, first we introduce $H=L^{2}[0, \pi]$ and $A_{0}: \mathcal{D}\left(A_{0}\right) \rightarrow H$ by

$$
\mathcal{D}\left(A_{0}\right)=\mathcal{H}^{2}(0, \pi) \cap \mathcal{H}_{0}^{1}(0, \pi), \quad A_{0} w=-w_{x x} .
$$

Then $A_{0}$ is a strictly positive operator, so that for every $\alpha>0$ we can introduce the space $H_{\alpha}=\mathcal{D}\left(A_{0}^{\alpha}\right)$, with $\|z\|_{\alpha}=\left\|A_{0}^{\alpha} z\right\|_{H}$. We set $H_{0}=H$ and $H_{-\alpha}=H_{\alpha}^{\prime}$ (duality with respect to the pivot space $H)$. We have

$$
H_{\frac{1}{2}}=\mathcal{H}_{0}^{1}(0, \pi),
$$

see for instance [20, Section 3.5]. The semigroup $\mathbb{T}$ associated to our PDE is defined on the state space $X=H_{\frac{1}{2}} \times H$, with $X_{1}=\mathcal{D}(A)=H_{1} \times H_{\frac{1}{2}}$ and

$$
A\left[\begin{array}{l}
g \\
f
\end{array}\right]=\left[\begin{array}{c}
f \\
-A_{0} g
\end{array}\right] \text {. }
$$

This $A$ is skew-adjoint and hence $\mathbb{T}$ is unitary. We have $Y=\mathbb{C}^{2}$ and the observation operator $C \in \mathcal{L}\left(X_{1}, Y\right)$ is given by

$$
C\left[\begin{array}{l}
g \\
f
\end{array}\right]=\left[\begin{array}{c}
-g_{x}(0) \\
g_{x}(\pi)
\end{array}\right] \quad \forall\left[\begin{array}{l}
g \\
f
\end{array}\right] \in H_{1} \times H_{\frac{1}{2}} .
$$

The PDE can be solved by elementary methods and the corresponding semigroup is isomorphic to a periodic left shift semigroup on $[0,2 \pi]$, see for instance Weiss [22, Section $5]$. However, in order to express $\Psi_{\tau}$ for small $\tau$, we do not need the solution formulas for the system (3.6), (3.7). It will be enough to recall the elementary fact that if $w$ is a solution of (3.6),(3.7) then for $x \in[0, \pi]$ and $\delta \in \mathbb{R}$ such that $x+\delta \in[0, \pi]$ we have

$$
\dot{w}(x, t)-w_{x}(x, t)=\dot{w}(x+\delta, t+\delta)-w_{x}(x+\delta, t+\delta) .
$$

Similarly, if $x \in[0, \pi]$ and $\delta \in \mathbb{R}$ are such that $x-\delta \in[0, \pi]$, we have

$$
\dot{w}(x, t)+w_{x}(x, t)=\dot{w}(x-\delta, t+\delta)+w_{x}(x-\delta, t+\delta) .
$$

The expression in the last two formulas are called Riemann invariants of the wave equation and these formulas say that they are constant along characteristics.

If we take $x=\pi, t \in[0, \pi]$ and $\delta=-t$ in (3.8), we get

$$
w_{x}(\pi, t)=w_{x}(\pi-t, 0)-\dot{w}(\pi-t, 0)=w_{0, x}(\pi-t)-w_{1}(\pi-t) .
$$

Similarly, if we take $x=0, t \in[0, \pi]$ and $\delta=-t$ in (3.9), we get

$$
w_{x}(0, t)=w_{x}(t, 0)+\dot{w}(t, 0)=w_{0, x}(t)+w_{1}(t) .
$$


As usual, we denote by $\Psi_{\tau}$ the output maps corresponding to the pair $(A, C)$. From the last two formulas, denoting $g=w_{0}$ and $f=w_{1}$, it follows that

$$
\Psi_{\pi}\left[\begin{array}{l}
g \\
f
\end{array}\right]=\left[\begin{array}{c}
-g_{x}-f \\
\boldsymbol{Я}_{\pi}\left(g_{x}-f\right)
\end{array}\right] \quad \forall\left[\begin{array}{l}
g \\
f
\end{array}\right] \in X .
$$

This shows, in particular, that $(A, C)$ is exactly observable in time $\pi$, because both $g_{x}$ and $f$ can be continuously recovered from $g_{x}+f$ and from $g_{x}-f$.

Sometimes (such as in the example discussed in Section 5) we are interested to know if it is possible to recover an initial state of the special structure $\left[\begin{array}{l}0 \\ f\end{array}\right]$, and then the equivalent question is if $\Psi_{\tau}$ is bounded from below on the subspace of such initial states. We can see from (3.10) that this is indeed the case for $\tau \geqslant \pi / 2$, i.e., if we know that the initial state has the special structure $\left[\begin{array}{l}0 \\ f\end{array}\right]$, then we need only half the time to recover it than we would otherwise need, see also Komornik and Yamamoto [10].

A simple computation shows that

$$
C^{*}=-\left[\begin{array}{l}
D \\
0
\end{array}\right]
$$

where $D: \mathbb{C}^{2} \rightarrow H$ is the Dirichlet map:

$$
D\left[\begin{array}{l}
u_{1} \\
u_{2}
\end{array}\right](x)=\left(1-\frac{x}{\pi}\right) u_{1}+\frac{x}{\pi} u_{2}
$$

and this easily implies that

$$
Z_{1}^{d}=X_{1}^{d}+A^{-1} C^{*} Y=\left(\mathcal{H}^{2}(0, \pi) \cap \mathcal{H}_{0}^{1}(0, \pi)\right) \times \mathcal{H}^{1}(0, \pi)
$$

(we have used that $X_{1}^{d}=\mathcal{D}\left(A^{*}\right)=\mathcal{D}(A)$, because $A$ is skew-adjoint). According to Proposition 3.1 with $m=1$, for every $\tau \geqslant 0, \Psi_{\tau}$ is bounded from $\left(Z_{1}^{d}\right)^{\prime}$ to $\left[\mathcal{H}_{R}^{1}\left(0, \tau ; \mathbb{C}^{2}\right)\right]^{\prime}$, and for $\tau>\pi$, according to Theorem 3.2, this operator is bounded from below.

Remark 3.4. In this remark we signal what we think to be a small mistake in two papers of Komornik and Yamamoto [10, 12], appearing also in Cipolatti and Yamamoto [4], where a certain inequality seems to contradict our Proposition 3.1.

In Section 4 of [12] the authors study the wave equation on an $n$-dimensional ball $\Omega$, with Neumann boundary observation over the whole boundary. Let us denote $A_{0}=-\Delta$, $\mathcal{D}\left(A_{0}\right)=\mathcal{H}^{2}(\Omega) \cap \mathcal{H}_{0}^{1}(\Omega)$, the spaces $H_{\alpha}$ (with $\alpha \in \mathbb{R}$ ) are defined as for the one dimensional case above and $Y=L^{2}(\partial \Omega)$. In [12, Proposition 4.2] it is claimed that the output maps $\Psi_{\tau}$ of this system can be extended so that they boundedly map initial states of the form $\left[\begin{array}{l}0 \\ f\end{array}\right]$ with $f \in H_{-\frac{s}{2}}(s \geqslant 0)$ into $\left[\mathcal{H}_{R}^{s}(0, \tau ; Y)\right]^{\prime}$ (which is defined by interpolation). This boundedness is expressed as the existence of $c>0$ such that

$$
\left\|\Psi_{\tau}\left[\begin{array}{l}
0 \\
f
\end{array}\right]\right\|_{\left[\mathcal{H}_{R}^{s}(0, \tau ; Y)\right]^{\prime}} \leqslant c\|f\|_{H_{-\frac{s}{2}}}
$$

(this is the second half of [12, estimate (4.3)]). (Actually, this is claimed only for $\tau$ larger than the radius of the ball, but if it were true, then this would easily imply that it is true for any $\tau \geqslant 0$.) Moreover, it is claimed in the same place that if $\tau$ is larger than the radius of the ball, then this map is bounded from below. This result (the boundedness part) contradicts our Proposition 3.1, because it would mean that (at least in the given 
context) $\Psi_{\tau}$ boundedly maps $X_{-s}$ into $\left[\mathcal{H}_{R}^{s}(0, \tau ; Y)\right]^{\prime}$. It is claimed in [4, Proposition 2.2] that the same result generalizes to any bounded open set $\Omega$ with smooth boundary.

To clarify if there is indeed a mistake, we consider the one dimensional domain $\Omega=(0, \pi)$ and we take $s=1$ and $\tau=\pi$, so that we can use our computations from Example 3.3. Then (3.11) with the help of (3.10) reduces to

$$
\left\|\left[\begin{array}{c}
f \\
\boldsymbol{G}_{\pi} f
\end{array}\right]\right\|_{\left[\mathcal{H}_{R}^{1}(0, \pi ; Y)\right]^{\prime}} \leqslant c\|f\|_{\mathcal{H}^{-1}(0, \pi)},
$$

which would imply in particular that

$$
\|f\|_{\left[\mathcal{H}_{R}^{1}(0, \pi)\right]^{\prime}} \leqslant c\|f\|_{\mathcal{H}^{-1}(0, \pi)} \quad \forall f \in L^{2}[0, \pi] .
$$

To disprove (3.12) we construct a sequence $\left(f_{n}\right)$ of functions in $L^{2}[0, \pi]$ such that

$$
\left\|f_{n}\right\|_{\mathcal{H}^{-1}(0, \pi)} \rightarrow 0, \quad\left\|f_{n}\right\|_{\left[\mathcal{H}_{R}^{1}(0, \pi)\right]^{\prime}} \nrightarrow 0 .
$$

Such a sequence is given by

$$
f_{n}(x)=n e^{-n x} .
$$

We have (using the duality pairing between $\mathcal{H}^{-1}(0, \pi)$ and $\left.\mathcal{H}_{0}^{1}(0, \pi)\right)$

$$
\left\|f_{n}\right\|_{\mathcal{H}^{-1}(0, \pi)}=\sup _{\varphi \in \mathcal{H}_{0}^{1}(0, \pi),\left\|\varphi^{\prime}\right\|_{L^{2}}=1}\left|\left\langle f_{n}, \varphi\right\rangle\right|=\sup _{\varphi \in \mathcal{H}_{0}^{1}(0, \pi),\left\|\varphi^{\prime}\right\|_{L^{2}}=1}\left|\int_{0}^{\pi} e^{-n x} \varphi^{\prime}(x) \mathrm{d} x\right| .
$$

Now we see from the Cauchy-Schwarz inequality that indeed $\left\|f_{n}\right\|_{\mathcal{H}^{-1}(0, \pi)} \rightarrow 0$. Finally, to show that $\left(f_{n}\right)$ does not converge to zero in $\left[\mathcal{H}_{R}^{1}(0, \pi)\right]^{\prime}$, we take $\varphi \in \mathcal{H}_{R}^{1}(0, \pi)$ defined by $\varphi(x)=\pi-x$. Then we have the duality pairing

$$
\left\langle f_{n}, \varphi\right\rangle=-\left[e^{-n x} \varphi(x)\right]_{0}^{\pi}+\int_{0}^{\pi} e^{-n x}(-1) \mathrm{d} x=\pi-\frac{1-e^{-n \pi}}{n} \rightarrow \pi .
$$

The same problem appears in the discussion of the one dimensional case in [10]. Indeed, the first part of the estimate (12) from [10] is $(3.12)$, with the interval $(0, \pi)$ replaced with $(0,1)$, both in space and in time. The proof of this estimate given in [10] (with an erratum given in [11]) is valid for $f \in F$, where $F$ is a certain dense subspace of $\mathcal{H}^{-1}(0, \pi)$. The mistake is to conclude from here that the estimate holds for $f \in\left[\mathcal{H}_{R}^{1}(0, \pi)\right]^{\prime}$, because $F$ is not dense in $\left[\mathcal{H}_{R}^{1}(0, \pi)\right]^{\prime}$.

\section{The main result}

In this section we give our abstract result regarding the identification of source terms. Let $X$ and $Y$ be Hilbert spaces. We consider systems of the type

$$
\begin{aligned}
& \dot{z}(t)=A z(t)+\lambda(t) f, \quad z(0)=0, \\
& y(t)=C z(t)
\end{aligned}
$$

where $A$ is the generator of a strongly continuous semigroup $\mathbb{T}$ on $X, C \in \mathcal{L}\left(X_{1}, Y\right)$ is an admissible observation operator for $\mathbb{T}$ and $(A, C)$ is exactly observable in time $\tau_{0}$. 
If $f \in X$ and $\lambda$ is a continuous (scalar) function, then it is easy to see that the solution of (4.1) on any interval $[0, \tau]$ gives the following output function:

$$
y(t)=\left(\mathcal{J}_{\tau} f\right)(t)=\int_{0}^{t} \lambda(t-s)\left(\Psi_{\tau} f\right)(s) \mathrm{d} s \quad \forall t \in[0, \tau],
$$

where $\Psi_{\tau}$ is defined as at the beginning of Section 3 .

Here we are concerned with extensions of this operator $\mathcal{J}_{\tau}$ (from $f$ to $y$ ) to some larger spaces. For $m \in \mathbb{N}$, the spaces $X_{m}, X_{-m}, X_{m}^{d}$ and $X_{-m}^{d}$ are defined as at the beginning of Section 2. In our main result we consider $f \in\left(Z_{m}^{d}\right)^{\prime}$ (these extended spaces were introduced in the previous section) and $\lambda \in W^{1,1}(0, \tau)$ (defined before Theorem 1.1). We may regard $f$ as a control operator and $\lambda$ as an input function, in the sense of [20], but we do not assume that $f$ is an admissible control operator for $\mathbb{T}$. We do not even assume that $f \in X_{-1}$, we only have that $f \in X_{-m}$ (because $\left(Z_{m}^{d}\right)^{\prime} \subset X_{-m}$ ). Systems with a control operator mapping into $X_{-m}$ have been considered, for instance, in Latushkin et al [14].

Lemma 4.1. Let $\tau>0, k \in L^{1}[0, \tau]$ and define the operator $C_{k} \in \mathcal{L}\left(L^{2}([0, \tau] ; Y)\right)$ by

$$
\left(C_{k} v\right)(t)=\int_{0}^{t} k(t-s) v(s) \mathrm{d} s .
$$

Then $\sigma\left(C_{k}\right)=\{0\}$.

Proof. It is well known that $\left\|C_{k}\right\| \leqslant\|k\|_{L^{1}}$. For every $\gamma \in \mathbb{R}$ we define the operator $e_{\gamma} \in \mathcal{L}\left(L^{1}[0, \tau]\right)$ by $\left(e_{\gamma} v\right)(t)=e^{\gamma t} v(t)$. It easy to check that

$$
C_{k}=e_{-\gamma} C_{e_{\gamma} k} e_{\gamma}
$$

which shows that for any nonzero $\alpha \in \mathbb{C}, \alpha I-C_{k}$ is invertible iff $\alpha I-C_{e_{\gamma} k}$ is invertible. According to the dominated convergence theorem we have $\lim _{\gamma \rightarrow-\infty}\left\|e_{\gamma} k\right\|_{L^{1}}=0$, so that $\left\|C_{e_{\gamma} k}\right\| \rightarrow 0$ as $\gamma \rightarrow-\infty$. This implies that indeed $\alpha I-C_{e_{\gamma} k}$ is invertible.

For results related to the above lemma we refer to Gohberg and Feldman [9, p. 41, p. 100] or Kress [13, p. 33, p. 34].

Remark 4.2. It is easy to see that $C_{k}$ from the last lemma commutes with the operator of integration from 0 to $t$. This fact together with the lemma implies that for any $\alpha \in \mathbb{C} \backslash\{0\}$ and $m \in \mathbb{N}, \alpha I-C_{k}$ is an isomorphism (i.e., it is boundedly invertible) on $\mathcal{H}_{L}^{m}(0, \tau ; Y)$.

Lemma 4.3. Let $\tau>0$ and $\lambda \in W^{1,1}(0, \tau)$ with $\lambda(0) \neq 0$. Define $S_{\tau}: L^{2}([0, \tau] ; Y) \rightarrow$ $\mathcal{H}_{L}^{1}(0, \tau ; Y)$ by

$$
\left(S_{\tau} g\right)(t)=\int_{0}^{t} \lambda(t-s) g(s) \mathrm{d} s .
$$

Then $S_{\tau}$ is an isomorphism from $L^{2}([0, \tau] ; Y)$ onto $\mathcal{H}_{L}^{1}(0, \tau ; Y)$. Moreover, for every $m \in$ $\mathbb{N}$, the operator $S_{\tau}$ admits a unique extension to an isomorphism

$$
S_{\tau}:\left[\mathcal{H}_{R}^{m}(0, \tau ; Y)\right]^{\prime} \rightarrow\left[\mathcal{H}_{R}^{m-1}(0, \tau ; Y)\right]^{\prime} .
$$

Proof. Denote $y=S_{\tau} g$. Differentiating the sides of (4.3) we obtain that

$$
\dot{y}(t)=\lambda(0) g(t)+\int_{0}^{t} \dot{\lambda}(t-s) g(s) \mathrm{d} s .
$$


Thus, the operator from $g$ to $\dot{y}$ is $\lambda(0) I+C_{k}$, with $k=\dot{\lambda}$. According to Lemma 4.1 this operator is an isomorphism on $L^{2}([0, \tau] ; Y)$. Since $y(t)=\int_{0}^{t} \dot{y}(\sigma) \mathrm{d} \sigma$, it follows that $S_{\tau}$ is an isomorphism from $L^{2}([0, \tau] ; Y)$ onto $\mathcal{H}_{L}^{1}(0, \tau ; Y)$.

Denote $\mathcal{X}=L^{2}([0, \tau] ; Y), \mathcal{D}(\mathcal{A})=\mathcal{H}_{L}^{1}(0, \tau ; Y)$ and let $\mathcal{A}: \mathcal{D}(\mathcal{A}) \rightarrow \mathcal{X}$ be the inverse of $S_{\tau}$. For $m \in \mathbb{N}$ we denote by $\mathcal{X}_{m}$ the space $\mathcal{D}\left(\mathcal{A}^{m}\right)$ endowed with the graph norm. It is not difficult to check (using Remark 4.2) that

$$
\mathcal{X}_{m}=\mathcal{H}_{L}^{m}(0, \tau ; Y) \quad \forall m \in \mathbb{N} .
$$

The spaces $\mathcal{X}_{-m}$, with $m \in \mathbb{N}$ are defined as the completion of $\mathcal{X}$ with respect to the norm $\|z\|_{-m}=\left\|(\beta I-\mathcal{A})^{-m} z\right\|$, where $\beta \in \rho(\mathcal{A})$. We refer to [20, Section 2.10] for a detailed discussion of such spaces. In particular, it is well known that $\mathcal{X}_{-m}$ is the dual of $\mathcal{D}\left(\left(\mathcal{A}^{*}\right)^{m}\right)$ with respect to the pivot space $\mathcal{X}$. It is easy to check that

$$
\left(S_{\tau}^{*} h\right)(t)=\int_{t}^{\tau} \bar{\lambda}(s-t) h(s) \mathrm{d} s,
$$

so that $S_{\tau}^{*}$ maps $L^{2}([0, \tau] ; Y)$ onto $\mathcal{H}_{R}^{1}(0, \tau ; Y)$, whence $\mathcal{D}\left(\mathcal{A}^{*}\right)=\mathcal{H}_{R}^{1}(0, \tau ; Y)$. More generally, by the dual version of Remark $4.2, \mathcal{D}\left(\left(\mathcal{A}^{*}\right)^{m}\right)=\mathcal{H}_{R}^{m}(0, \tau ; Y)$, so that we have

$$
\mathcal{X}_{-m}=\left[\mathcal{H}_{R}^{m}(0, \tau ; Y)\right]^{\prime} \quad \forall m \in \mathbb{N} .
$$

By denoting $\mathcal{X}_{0}=\mathcal{X}, \mathcal{A}$ maps $\mathcal{X}_{m}$ onto $\mathcal{X}_{m-1}$ for every $m \in \mathbb{Z}$. Consequently, $S_{\tau}=\mathcal{A}^{-1}$ extends to an isomorphism from $\left[\mathcal{H}_{R}^{m}(0, \tau ; Y)\right]^{\prime}$ onto $\left[\mathcal{H}_{R}^{m-1}(0, \tau ; Y)\right]^{\prime}$.

Theorem 4.4. Assume that the pair $(A, C)$ is exactly observable in some time $\tau_{0}>0$ and that $\lambda \in W^{1,1}(0, \tau)$ with $\lambda(0) \neq 0$. Then for every $m \in\{0,1,2, \ldots\}$ and each $\tau>\tau_{0}, \mathcal{J}_{\tau}=$ $S_{\tau} \Psi_{\tau}$ is one-to-one from $\left(Z_{m}^{d}\right)^{\prime}$ to $\left[\mathcal{H}_{R}^{m-1}(0, \tau ; Y)\right]^{\prime}$ and there exists a constant $\kappa_{m, \tau}>0$ such that

$$
\|f\|_{\left(Z_{m}^{d}\right)^{\prime}} \leqslant \kappa_{m, \tau}\left\|\mathcal{J}_{\tau} f\right\|_{\left[\mathcal{H}_{R}^{m-1}(0, \tau ; Y)\right]^{\prime}}, \quad \forall f \in\left(Z_{m}^{d}\right)^{\prime} .
$$

Proof. By using Lemma 4.3 and Theorem 3.2, there exists $M_{\tau}>0$ such that

$$
\left\|\mathcal{J}_{\tau} f\right\|_{\left[\mathcal{H}_{R}^{m-1}(0, \tau ; Y)\right]^{\prime}} \geqslant M_{\tau}\left\|\Psi_{\tau} f\right\|_{\left[\mathcal{H}_{R}^{m}(0, \tau ; Y)\right]^{\prime}} \geqslant M_{\tau} c_{m, \tau}\|f\|_{\left[Z_{m}^{d}\right]^{\prime}}
$$

Remark 4.5. The above theorem considers $f \in\left(Z_{m}^{d}\right)^{\prime}$. This implies, according to the definition of $Z_{m}^{d}$, that the duality products $\left\langle f,\left(s I-A^{*}\right)^{-k} C^{*} y_{0}\right\rangle$ are well defined for all $y_{0} \in Y$ and all $k \in\{1,2, \ldots m\}$, so that the products $f^{*}\left(s I-A^{*}\right)^{-k} C^{*}$ make sense.

\section{An application to the wave equation}

Consider the system described by (1.1), with the stated assumptions on $\Omega, \Gamma$ and $\lambda$. Our aim here is to prove Theorem 1.1, which shows that the inverse problem of determining the location of the source $\xi$ from the output $y$, is well-posed in appropriate spaces. Note that Theorem 1.1 implies the existence of $K>0$ such that for any $\xi^{(1)}, \xi^{(2)} \in \Omega_{\varepsilon}$,

$$
\left\|y^{(1)}-y^{(2)}\right\|_{L^{2}\left(0, \tau ; L^{2}(\Gamma)\right)} \geqslant K\left|\xi^{(1)}-\xi^{(2)}\right|,
$$

where the $L^{2}$ norm on the left might be $\infty$. 
For the proof we need to introduce some notation. We denote by $-A_{0}$ the Dirichlet Laplacian on $\Omega$, so that $A_{0}: \mathcal{D}\left(A_{0}\right) \rightarrow L^{2}(\Omega)$, where

$$
\mathcal{D}\left(A_{0}\right)=\mathcal{H}^{2}(\Omega) \cap \mathcal{H}_{0}^{1}(\Omega),
$$

and $A_{0}>0$. We denote $H=L^{2}(\Omega)$ and for every $\alpha>0$ we define $H_{\alpha}=\mathcal{D}\left(A_{0}^{\alpha}\right)$, with the norm $\|z\|_{\alpha}=\left\|A_{0}^{\alpha} z\right\|_{H}$. The spaces $H_{-\alpha}$ are defined as the duals of $H_{\alpha}$ with respect to the pivot space $H$. We have (see, for instance, Section 3.6 in [20])

$$
H_{1}=\mathcal{H}^{2}(\Omega) \cap \mathcal{H}_{0}^{1}(\Omega), \quad H_{\frac{1}{2}}=\mathcal{H}_{0}^{1}(\Omega), \quad H_{-\frac{1}{2}}=\mathcal{H}^{-1}(\Omega) .
$$

We recall the following well-known result defining the Dirichlet map $D$ for $\Omega$ (see, for instance, Propositions 10.6.1 and 10.6.2 in [20]).

Proposition 5.1. For every $\mathrm{v} \in L^{2}(\partial \Omega)$, there exists a unique function $D \mathrm{v} \in L^{2}(\Omega)$ such that

$$
\int_{\Omega}(D \mathrm{v})(x) g(x) \mathrm{d} x=-\int_{\partial \Omega} \mathrm{v} \frac{\partial\left(A_{0}^{-1} g\right)}{\partial \nu} \mathrm{d} \sigma \quad \forall g \in L^{2}(\Omega) .
$$

The operator $D$ defined above (called the Dirichlet map) is linear and bounded from $L^{2}(\partial \Omega)$ into $L^{2}(\Omega)$ and its adjoint $D^{*} \in \mathcal{L}\left(L^{2}(\Omega), L^{2}(\partial \Omega)\right)$ given by

$$
D^{*} g=-\frac{\partial\left(A_{0}^{-1} g\right)}{\partial \nu} \quad \forall g \in L^{2}(\Omega) .
$$

Moreover, for any $\mathrm{v} \in L^{2}(\partial \Omega)$ we have $D \mathrm{v} \in C^{\infty}(\Omega)$ and $\Delta D \mathrm{v}=0$.

We mention that $\gamma_{0} D \mathrm{v}=\mathrm{v}$ for all $\mathrm{v} \in L^{2}(\partial \Omega)$, where $\gamma_{0}$ is a suitable extension of the Dirichlet trace operator, see for instance [20, Proposition 10.6.4].

The system (1.1) can be rewritten in the abstract form (4.1), with the following spaces and operators:

$$
\begin{array}{rlrl}
X=H_{\frac{1}{2}} \times H, & Y & =L^{2}(\Gamma), \\
\mathcal{D}(A)=H_{1} \times H_{\frac{1}{2}}, & A & =\left[\begin{array}{cc}
0 & I \\
-A_{0} & 0
\end{array}\right] \\
C & =\left[\begin{array}{ll}
\gamma_{1} & 0
\end{array}\right], & f & =\left[\begin{array}{c}
0 \\
\delta_{\xi}
\end{array}\right],
\end{array}
$$

where $\gamma_{1}$ is the Neumann trace operator restricted to $\Gamma$. Note that $A^{-1}=\left[\begin{array}{cc}0 & -A_{0}^{-1} \\ I & 0\end{array}\right]$.

Lemma 5.2. For every $\xi \in \Omega$ and $m>n / 2$, we have $f \in\left(Z_{m}^{d}\right)^{\prime}$.

Proof. A simple calculation based on (5.2) shows that $C^{*}=-\left[\begin{array}{l}D \\ 0\end{array}\right]$, where $D$ is the Dirichlet map defined in Proposition 5.1. Since $A$ is skew-adjoint, we have

$$
X_{m}^{d}=H_{\frac{m+1}{2}} \times H_{\frac{m}{2}} .
$$

Hence, by a short computation, the space $Z_{m}^{d}$ from (3.2) (using $\beta=0$ ) is given by

$$
Z_{m}^{d}=X_{m}^{d}+A^{-1} C^{*} Y+A^{-2} C^{*} Y \ldots+A^{-m} C^{*} Y=W_{m} \times V_{m},
$$


where

$$
\begin{gathered}
W_{m}=H_{\frac{m+1}{2}}+A_{0}^{-1} D Y \ldots+A_{0}^{-[(m+1) / 2]} D Y, \\
V_{m}=H_{\frac{m}{2}}+A_{0}^{-1} D Y \ldots+A_{0}^{-[m / 2]} D Y,
\end{gathered}
$$

and $[\alpha]$ denotes the integer part of the real number $\alpha$.

We claim that for $m>n / 2, V_{m} \subset C(\Omega)$. Indeed, if $\varphi \in V_{m}$ then its first component according to the above decomposition is in $H_{\frac{m}{2}} \subset \mathcal{H}^{m}(\Omega) \subset C(\Omega)$ (we have used a well known Sobolev embedding theorem). The other components of $\varphi$ are in $A_{0}^{-k} D Y$, for various $k \in \mathbb{N}$. We know from Proposition 5.1 that $D Y \subset C^{\infty}(\Omega)$. According to a regularity result for elliptic equations (see Evans [8, p. 309]), $A_{0}^{-k} D Y \subset C^{\infty}(\Omega)$.

We claim that for every $\varepsilon>0$ and for $m>n / 2$, the operator $T_{\varepsilon}: V_{m} \rightarrow C\left(\bar{\Omega}_{\varepsilon}\right)$ defined by

$$
T_{\varepsilon} \varphi=\left.\varphi\right|_{\bar{\Omega}_{\varepsilon}}
$$

is bounded. (On $C\left(\bar{\Omega}_{\varepsilon}\right)$ we use the supremum norm.) Indeed, since $V_{m}$ is continuously embedded in $H$, it follows that $T_{\varepsilon}$ is bounded from $V_{m}$ to the larger space $L^{2}\left(\Omega_{\varepsilon}\right)$. Using the closed graph theorem, the boundedness of $T_{\varepsilon}$ (to $C\left(\bar{\Omega}_{\varepsilon}\right)$ ) follows.

From the above claim it follows that for every $\xi \in \Omega$ and $m>n / 2$, the Dirac mass $\delta_{\xi}$ is a bounded functional on $V_{m}$. Indeed, if we choose $\varepsilon>0$ such that $\xi \in \Omega_{\varepsilon}$, then

$$
\left|\left\langle\delta_{\xi}, \varphi\right\rangle\right|=|\varphi(\xi)| \leqslant\left\|T_{\varepsilon}\right\| \cdot\|\varphi\|_{V_{m}} \quad \forall \varphi \in V_{m} .
$$

From here, the lemma follows.

Proof of Theorem 1.1. Let $\varepsilon>0, m>n / 2, \xi^{(1)}, \xi^{(2)} \in \Omega_{\varepsilon}, \tau>\tau_{0}$. For $j \in\{1,2\}$ let $y^{(j)}$ be the output of (1.1) corresponding to $\xi=\xi^{(j)}$, with the initial data $\left[\begin{array}{l}w_{0} \\ w_{1}\end{array}\right] \in X$. These outputs are defined by continuous extension: we approximate $\delta_{\xi^{(j)}}$ by a sequence of functions $\delta_{k, j} \in L^{2}(\Omega)$, with convergence in $V_{m}^{\prime}\left(V_{m}\right.$ was defined in (5.3)). Then from the decomposition $\left(Z_{m}^{d}\right)^{\prime}=W_{m}^{\prime} \times V_{m}^{\prime}$ (see the proof of Lemma 5.2) we see that $\left[\begin{array}{c}0 \\ \delta_{k, j}\end{array}\right] \rightarrow\left[\begin{array}{c}0 \\ \delta_{\xi^{(j)}}\end{array}\right]$ in $\left(Z_{m}^{d}\right)^{\prime}$. For $\delta_{k, j}$ in place of $\delta_{\xi^{(j)}}$, the output of $(1.1)$ on $[0, \tau]$ is

$$
y_{k, j}=\Psi_{\tau}\left[\begin{array}{l}
w_{0} \\
w_{1}
\end{array}\right]+\mathcal{J}_{\tau}\left[\begin{array}{c}
0 \\
\delta_{k, j}
\end{array}\right]
$$

where $\mathcal{J}_{\tau}$ is the operator introduced in (4.2), with our specific $A$ and $C$. According to Theorem 4.4 the operator $\mathcal{J}_{\tau}$ has a continuous extension from $\left(Z_{m}^{d}\right)^{\prime}$ to $\left[\mathcal{H}_{R}^{m-1}(0, \tau ; Y)\right]^{\prime}$. Thus, $y^{(j)}$ can be defined in the latter space as the limit of $y_{k, j}$.

Denoting $f^{(j)}=\left[\begin{array}{c}0 \\ \delta_{\xi_{j}}\end{array}\right]$, we obtain that

$$
y^{(j)}=\Psi_{\tau}\left[\begin{array}{l}
w_{0} \\
w_{1}
\end{array}\right]+\mathcal{J}_{\tau} f^{(j)} \in\left[\mathcal{H}_{R}^{m-1}(0, \tau ; Y)\right]^{\prime} .
$$

Subtracting these equations and using Theorem 4.4, we obtain that

$$
\left\|f^{(1)}-f^{(2)}\right\|_{\left(Z_{m}^{d}\right)^{\prime}} \leqslant \kappa_{m, \tau}\left\|y^{(1)}-y^{(2)}\right\|_{\left[\mathcal{H}_{R}^{m-1}(0, \tau ; Y)\right]^{\prime}} .
$$


To end the proof we note that for every $j \in\{1, \ldots, n\}$ there exists a function $\varphi_{j} \in$ $\mathcal{D}(\Omega) \subset V_{m}$ such that

$$
\varphi_{j}\left(x_{1}, x_{2}, \ldots x_{n}\right)=x_{j} \quad \forall\left(x_{1}, x_{2}, \ldots, x_{n}\right) \in \Omega_{\varepsilon}
$$

(this follows, for instance, from [20, Proposition 13.1.5]). Since $\xi^{(1)}, \xi^{(2)} \in \Omega_{\varepsilon}$, we have

$$
\left\langle\delta_{\xi^{(1)}}-\delta_{\xi^{(2)}}, \varphi_{j}\right\rangle=\xi_{j}^{(1)}-\xi_{j}^{(2)} .
$$

Thus, denoting $\eta_{m, \varepsilon}=\max _{j \in\{1,2, \ldots n\}}\left\|\varphi_{j}\right\|_{V_{m}}$, it follows that

$$
\left|\xi^{(1)}-\xi^{(2)}\right| \leqslant \sqrt{n} \eta_{m, \varepsilon}\left\|\delta_{\xi^{(1)}}-\delta_{\xi^{(2)}}\right\|_{V_{m}^{\prime}} .
$$

Since $\left\|f^{(j)}\right\|_{\left(Z_{m}^{d}\right)^{\prime}}=\left\|\delta_{\xi_{j}}\right\|_{V_{m}^{\prime}}$ and using (5.4), we get the desired estimate (1.2).

We mention that many interesting estimates related to (5.5) (also in the converse direction) can be found in Komornik and Yamamoto [12, Section 2].

\section{References}

[1] C. Alves, A.L. Silvestre, T. Takahashi and M. Tucsnak, Solving inverse source problems using observability. Applications to the Euler-Bernoulli plate equation, SIAM J. Control and Optim. 48 (2009), pp. 1632-1659.

[2] C. Bardos, G. Lebeau and J. Rauch, Sharp sufficient conditions for the observation, control and stabilization of waves from the boundary, SIAM J. Control and Optim. 30 (1992), pp. 1024-1065.

[3] N. Burq and P. Gérard, Condition nécessaire et suffisante pour la contrôlabilité exacte des ondes, C. R. Acad. Sci. Paris Sér. I Math. 325 (1997), pp. 749-752.

[4] R. Cipolatti and M. Yamamoto, An inverse problem for a wave equation with arbitrary initial values and a finite time of observations, Inverse Problems 27 (2011), 095006, 15 pages.

[5] B. Dehman and G. Lebeau, Analysis of the HUM control operator and exact controllability for semilinear waves in uniform time, SIAM J. Control and Optim. 48 (2009), pp. 521-550.

[6] A. El Badia and T. Ha-Duong, Determination of point wave sources by boundary measurements, Inverse Problems 17 (2001), pp. 1127-1139.

[7] S. Ervedoza and E. Zuazua, A systematic method for building smooth controls for smooth data, Discrete $\&$ Contin. Dynamical Systems Ser. B 14 (2010), pp. 1-27.

[8] L. C. Evans, Partial Differential Equations, vol. 19 of Graduate Studies in Mathematics, American Mathematical Society, Providence, RI, 1998.

[9] I. Gohberg and I. Feldman, Convolution Equations and Projection Methods for their Solution, American Math. Society, Translations of Math. Monographs Vol. 41, Providence, RI, 1974. 
[10] V. Komornik and M. Yamamoto, Upper and lower estimates in determining point sources in a wave equation, Inverse Problems 18 (2002), pp. 319-329.

[11] V. Komornik and M. Yamamoto, Corrigendum: "Upper and lower estimates in determining point sources in a wave equation", Inverse Problems 19 (2003), p. 999.

[12] V. Komornik and M. Yamamoto, Estimation of point sources and applications to inverse problems, Inverse Problems 21 (2005), pp. 2051-2070.

[13] R. Kress, Linear Integral Equations, vol. 82 of Applied Mathematical Sciences, Springer-Verlag, Berlin, 1989.

[14] Y. Latushkin, T. Randolph and R. Schnaubelt, Regularization and frequency domain stability pf well-posed systems, Math. of Control, Signals and Systems 17 (2005), pp. $128-151$.

[15] J.-L. Lions, Contrôlabilité exacte, perturbations et stabilisation de systèmes distribués. Tome 1, Recherches en Mathématiques Appliquées Vol. 8, Masson, Paris, 1988 (with a chapter by E. Zuazua, and a chapter by C. Bardos, G. Lebeau and J. Rauch).

[16] Y. Meyer, Étude d'un modèle mathématique issu du contrôle des structures spatiales déformables, in Nonlinear partial differential equations and their applications, Collège de France seminar Vol. VII, Paris 1983-1984, pp. 234-242, Res. Notes in Math. Vol. 122, Pitman, Boston, MA, 1985.

[17] J.-P. Puel and M. Yamamoto, Applications de la contrôlabilité exacte à quelques problèmes inverses hyperboliques, C. R. Acad. Sci. Paris Sér. I Math. 320 (1995), pp. 1171-1176.

[18] R. Triggiani, Interior and boundary regularity of the wave equation with interior point control, Differential Integral Equations 6 (1993), pp. 111-129.

[19] M. Tucsnak and G. Weiss, Simultaneous exact controllability and some applications, SIAM J. Control and Optim. 38 (2000), pp. 1408-1427.

[20] M. Tucsnak and G. Weiss, Observation and Control for Operator Semigroups, Birkhäuser Verlag, Basel, 2009.

[21] A.J. Weiss, Direct position determination of narrowband radio frequency transmitters, IEEE Signal Processing Letters 11 (2004), pp. 513-516.

[22] G. Weiss, Admissibility of unbounded control operators, SIAM J. Control and Optim. 27 (1989), pp. 527-545.

[23] G. Weiss, Admissible observation operators for linear semigroups, Israel J. Math. 65 (1989), pp. 17-43. 